\title{
Healthcare resource utilization after living liver donation: a retrospective case-control study
}

\author{
Hyunjae $\mathrm{Im}^{1}$, Ho Geol Ryu ${ }^{1}$, Eun Jin Jang ${ }^{2}$, Junwoo Jo ${ }^{3}$, Suk Hyung Choe ${ }^{1}$, Somin Joo ${ }^{1}$, Hannah Lee', Seung-Young $\mathrm{Oh}^{4}$, \\ Suk Kyun Hong ${ }^{4}$
}

\footnotetext{
${ }^{1}$ Department of Anesthesiology, Seoul National University Hospital, Seoul, Korea

${ }^{2}$ Department of Department of Information Statistics, Andong National University, Andong, Korea

${ }^{3}$ Department of Department of Statistics, Kyungpook National University, Daegu, Korea

${ }^{4}$ Department of Surgery, Seoul National University Hospital, Seoul, Korea
}

Background: Living liver donation is generally considered safe, but donors may experience short- or long-term complications. The purpose of this study was to assess healthcare resource utilization after liver donation in living liver donors in comparison to the general population.

Methods: Outpatient or emergency department visits and hospital admissions were compared between living liver donors who underwent hepatic resection for living liver donation between 2004 and 2018 and the matched general population. Healthcare resource utilization data for 5 years after liver donation were collected from the National Health Insurance Service (NHIS) database. For every living liver donor, four individually matched non-donors were selected from the NHIS database using age, sex, preexisting comorbidities, and previous healthcare utilization history.

Results: A total of 1,886 living liver donors and 7,309 non-donors were included. In the first year after donation, living liver donors required more outpatient department visits $(7[4-13]$ vs. $3[1-7], P<0.001)$ and more emergency department visits $(13.33 \%$ vs. $0.15 \%, P<0.001)$ compared to matched non-donors. A similar trend persisted for 5 years after donation. The number of hospital admissions of living liver donors was higher for up to 2 years after donation with longer hospital length of stay (13.0 [10.5-16.0] days vs. $5.0[3.0-9.0]$ days, $P<0.0001)$.

Conclusions: Healthcare resource utilization in living liver donors for 5 years after donation was higher compared to matched non-donors. The higher healthcare resource demand may be related to postoperative complications or lowered threshold for healthcare resource utilization after donation. 